\title{
Craneotomía guiada por ultrasonografía bidimensional para exéresis de tumor cerebral supratentorial
}

\author{
F. López-Hernández; J. Hernández-Palazón*; M. Reus-Pintado**; J.I. Garrido-Gómez* y J.F. Martínez-Lage
}

Servicios de Neurocirugía, Anestesiología y Reanimación* y Radiología**. Hospital Universitario Virgen de la Arrixaca. El Palmar. Murcia.

\section{Resumen}

Objetivos. El objetivo de este estudio descriptivo es presentar nuestra experiencia clínica durante un año sobre la utilización de la ultrasonografía bidimensional (2-D) en tiempo-real para la exéresis de tumor supratentorial de localización cerebral profunda.

Material y métodos. Durante el año 2006 realizamos craneotomía guiada por ultrasonografía 2-D en pacientes sometidos a cirugía para exéresis de tumor supratentorial, confirmado por anatomía patológica y de localización subcortical. Para la realización de la ultrasonografía se utilizaron los modelos de ultrasonidos M 2410 A ultrasounds HP y Phillips HDI 3500 ATL. Se utilizó en primer lugar un transductor de $3 \mathrm{Mhz}$ para localizar estructuras anatómicas, seguido de un transductor de $5 \mathrm{MHz}$ para la identificación del tumor cerebral. Se consiguieron cortes en el plano sagital y coronal durante la craneotomía parietal o temporal y en el plano axial y sagital en la craneotomía frontal. Tras la exéresis del tumor, se realizó una nueva ultrasonografía tras irrigar la cavidad con suero salino para la visualización de remanentes tumorales. Se realizó una tomografía computarizada (TC) craneal postoperatoria para verificar la exéresis total del tumor.

Resultados. Se incluyeron en el estudio 30 pacientes de edades comprendidas entre 28 y 82 años. El tiempo requerido para la localización de la lesión fue de 17,5 $( \pm 1,9)$ min. Se realizaron 8 craneotomías temporales, 7 parietooccipitales, 8 parietales, 2 frontoparietales y 5 parietotemporales. La lesión fue localizada a una profundidad media de 5,3 $( \pm 1,6) \mathrm{cm}$. El estudio histológico reveló 17 gliomas de alto grado, 3 gliomas de bajo grado, 8 lesiones metastásicas y 2 oligoastrocitomas. Los gliomas de alto grado mostraron mayor ecogenidad que los gliomas de bajo grado y el edema cerebral. No se detectaron restos del tumor al final de la cirugía, excepto en dos pacientes debido a la proximidad a áreas elocuentes del cerebro. La TC craneal postoperatoria

Recibido: 18-12-07. Aceptado: 2-05-08 confirmó la exéresis total del tumor en los pacientes incluidos en el estudio.

Conclusiones. La ultrasonografía 2-D en tiempo-real proporciona una imagen de calidad aceptable para la realización de craneotomía guiada para exéresis de tumores supratentoriales, ya que permite su localización de manera fiable y segura, sobre todo de tumores localizados en zonas del cerebro con gran importancia anatómica y funcional, así como identificar restos de tumor una vez finalizada la cirugía.

PALABRAS CLAVE: Tumor cerebral. Neurocirugía. Ultrasonidos bidimensional. Cirugía guiada por imagen.

Bidimensional-ultrasound guided-craniotomy in the excision of supratentorial brain tumours

\section{Summary}

Objectives. With this 1-year retrospective study we aimed to analyze our experience on the utilization of real-time bidimensional (2-D) ultrasonography as an aid for the localization of deep-seated supratentorial brain tumours.

Material and methods. During the year 2006 we performed 2-D ultrasonographic localization studies in patients undergoing excision of subcortical supratentorial brain tumours whose nature was pathologically proven. The ultrasound machines utilized were 2410 A ultrasounds HP and Phillips HDI 3500 ATL. To check gross anatomical structures we used a $3 \mathrm{MHz}$ probe and then a $5 \mathrm{MHz}$ sound to define the brain tumour characteristics. Coronal and sagittal cuts were obtained during parietal or temporal craniotomies, and axial and sagittal slices in the case of frontal masses. After brain tumour removal and after a thorough irrigation with saline, new image acquisitions were performed to confirm the completeness of tumour excision. The amount of removed tumour was also verified by a post-contrast computerized tomography (CT) scan performed after the surgery. 
Results. The study group was composed by 30 patients with ages comprised between 28 and 82 years. The time required for tumour localization was $\mathbf{1 7 . 5}$ $( \pm 1.9) \mathrm{min}$. The lesions were approached though 8 temporal, 7 parieto-occipital, 8 parietal, 2 fronto-parietal and 5 temporo-parietal craniotomies. Mean depth of the lesions was $5.3( \pm 1.6) \mathrm{cm}$. The histopathologic diagnosis was 17 high-grade gliomas, 3 low-grade astrocitomas, 8 metastatic lesions and 2 oligo-astrocytomas. Highgrade gliomas were more echogenic than low-grade tumours and than oedema. No residual tumour was detected at the end of the surgery except in two cases in which a tumoral rest was left due to the proximity of the lesion to eloquent cerebral areas. Postoperative CT scans confirmed the completeness of tumour removal in the patients of the study as assessed by intraoperative ultrasounds.

Conclusions. Real-time 2-D ultrasonography affords an adequate image quality for performing guided-craniotomy in supratentorial brain tumours, as it allows for a safe and accurate localization of the lesions. Its use is mainly indicated in the removal of tumours located in, or close to, important anatomical and functional brain areas and to depict the presence of tumour rests after surgical removal of cerebral neoplasms.

KEY WORDS: Brain tumour. Neurosurgery. Bidimensional ultrasonography. Image-guided surgery. Glioma. Brain metastasis.

\section{Introducción}

El objetivo de la neurocirugía en el tratamiento del tumor cerebral es conseguir la máxima exéresis del tejido patológico evitando la lesión del tejido cerebral sano que le circunda. Para alcanzar este objetivo, el neurocirujano dispone de diferentes métodos para el control intraoperatorio de la exéresis quirúrgica del tumor, que proporcionan información sobre las características, localización y demarcación de la lesión, así como el grado de resección realizado.

La ultrasonografía bidimensional (2-D) intraoperatoria (USIO) ha sido utilizada significativamente durante las últimas dos décadas para la obtención de imágenes en tiempo-real, siendo considerada el "gold standard" de los métodos de imagen utilizados para la orientación espacial intracraneal, debido a sus beneficios y fiabilidad durante la realización de diversos procedimientos neuroquirúrgicos ${ }^{2,5}$, 10,11,16. Los tumores cerebrales se pueden identificar como masa ecogénica, por lo que se ha utilizado la ultrasonografía 2-D como guía durante el procedimiento quirúrgico para exéresis de tumor cerebral ${ }^{3}$. La utilización de la USIO permite identificar con alta sensibilidad el tumor cerebral y diferenciarlo de las estructuras cerebrales normales durante la cirugía exerética, así como mostrar la vascularización relacionada con el tumor. También, la ultrasonografía 2$\mathrm{D}$ ofrece una modalidad de imagen en tiempo-real que ha sido utilizada con éxito como orientación en diferentes procedimientos neuroquirúrgicos como la realización de biopsia cerebral, colocación de catéter ventricular o drenaje de tumores quísticos 9 . En la actualidad, el desarrollo de sondas de ultrasonidos con mayor resolución, así como la existencia de un sistema color-duplex para la obtención de imágenes de alta calidad, han dotado a la ultrasonografía 2-D de una alta fiabilidad y de una relación señal-ruido comparable con las imágenes obtenidas por tomografía computarizada (TC) o resonancia magnética (RM), que ha permitido que la ultrasonografía 2-D se utilice cada vez con más frecuencia para el control de la resección radical de un tumor cerebral, ya que mejora la definición de la interfase tumor-cerebro además de detectar con elevada precisión la presencia de tumor residual ${ }^{9}$.

El objetivo de este estudio descriptivo es presentar nuestra experiencia clínica durante un año sobre la factibilidad y fiabilidad de la utilización de la ultrasonografía 2-D en tiempo-real para la exéresis de tumor supratentorial de localización cerebral profunda.

\section{Pacientes y métodos}

Durante el año 2006 realizamos craneotomía guiada por medio de ultrasonografía 2-D en 30 pacientes sometidos a cirugía electiva para exéresis de tumor supratentorial. Todos los pacientes fueron informados del procedimiento y dieron su consentimiento escrito, siendo aprobado por el Comité de Ética Asistencial del hospital. Las principales ventajas que valoraron los pacientes y cirujano sobre la aplicación de los ultrasonidos durante la craneotomía fueron la disminución del riesgo de lesión del tejido cerebral normal y facilitar la exéresis máxima del tumor. Los criterios de selección se aplicaron en relación con el tamaño y profundidad del tumor. Los tumores presentaron un tamaño entre 1 y $7 \mathrm{~cm}$ de diámetro y fueron localizados en la región supratentorial a nivel subcortical. Todas las intervenciones fueron realizadas por el mismo neurocirujano, así como el equipo de ultrasonografía fue utilizado por el mismo neurorradiólogo.

Para conseguir una imagen de calidad, se colocó a los pacientes en una posición para la craneotomía que permitiera que el área de la lesión cerebral se encuentre a menos de $7 \mathrm{~cm}$ del transductor de ultrasonografía. Tras la realización de la craneotomía y apertura de la duramadre, se impregnó la superficie cerebral con salino para mejorar la interfase antes de la aplicación del transductor de ultrasonidos, ejerciendo como acoplador acústico. Posteriormente, se aplicó crema conductora al transductor de ultrasonidos antes de recubrirlo con una cubierta estéril 
Tabla 1

Tiempo de localización, tamaño y profundidad del tumor

\begin{tabular}{lc}
\hline Tiempo localización tumor $(\mathrm{min})$ & $17,5( \pm 1,9 ; 15-20)$ \\
Tamaño tumor $(\mathrm{cm})$ & $4,5( \pm 1,9 ; 1-7)$ \\
Profundidad tumor $(\mathrm{cm})$ & $5,3( \pm 1,6 ; 3-8)$ \\
\hline
\end{tabular}

$\overline{\text { Los datos se muestran como media( } \pm \text { desviación estándar; rango) }}$ de plástico. A continuación se aplicó el transductor sobre la corteza cerebral intentado localizar la lesión tumoral mediante rotación y deslizamiento del mismo.

Para la realización de la USIO se recurrieron a los equipos de ultrasonografía $M 2410$ A ultrasounds HP y Phillips HDI 3500 ATL. Se utilizó en primer lugar un transductor de $3 \mathrm{MHz}$ de frecuencia para localizar estructuras anatómicas, seguido de uno de $5 \mathrm{MHz}$ de frecuencia para la identifica-

Tabla 2

Características demográficas de los pacientes estudiados, histología y localización del tumor

\begin{tabular}{|c|c|c|c|c|}
\hline Caso & Sexo & Edad & Diagnóstico & Localización \\
\hline 1 & $\mathrm{H}$ & 57 & Glioma alto grado & Parietooccipital derecho \\
\hline 2 & $\mathrm{H}$ & 71 & Glioma alto grado & Parietal derecho \\
\hline 3 & $\mathrm{H}$ & 54 & Metástasis adenocarcinoma & Frontoparietal derecho \\
\hline 4 & M & 61 & Glioma alto grado & Parietooccipital izquierdo \\
\hline 5 & $\mathrm{H}$ & 79 & Metástasis adenocarcinoma & Parietal izquierdo \\
\hline 6 & $\mathrm{H}$ & 54 & Glioma bajo grado & Temporal derecho \\
\hline 7 & $\mathrm{H}$ & 76 & Metástasis adenocarcinoma & Parietal izquierdo \\
\hline 8 & M & 69 & Glioma alto grado & Parietal izquierdo \\
\hline 9 & M & 58 & Glioma bajo grado & Parietotemporal derecho \\
\hline 10 & $\mathrm{H}$ & 71 & Metástasis adenocarcinoma & Frontoparietal derecho \\
\hline 11 & $\mathrm{H}$ & 64 & Glioma alto grado & Parietooccipital derecho \\
\hline 12 & $\mathrm{H}$ & 77 & Glioma bajo grado & Parietotemporal derecho \\
\hline 13 & $\mathrm{H}$ & 73 & Oligoastrocitoma & Parietotemporal izquierdo \\
\hline 14 & M & 48 & Glioma bajo grado & Parietal derecho \\
\hline 15 & $\mathrm{H}$ & 78 & Metástasis adenocarcinoma & Parietal derecho \\
\hline 16 & $\mathrm{H}$ & 76 & Glioma alto grado & Parietal izquierdo \\
\hline 17 & $\mathrm{H}$ & 65 & Glioma alto grado & Parietooccipital derecho \\
\hline 18 & $\mathrm{H}$ & 57 & Oligoastrocitoma & Parietooccipital izquierdo \\
\hline 19 & M & 58 & Glioma alto grado & Temporal derecho \\
\hline 20 & M & 63 & Glioma alto grado & Parietotemporal derecho \\
\hline 21 & $\mathrm{H}$ & 45 & Metástasis adenocarcinoma & Temporal izquierdo \\
\hline 22 & $\mathrm{H}$ & 65 & Glioma alto grado & Temporal izquierdo \\
\hline 23 & $\mathrm{H}$ & 73 & Metástasis adenocarcinoma & Frontotemporal derecho \\
\hline 24 & M & 82 & Glioma alto grado & Parietal izquierdo \\
\hline 25 & $\mathrm{H}$ & 66 & Metástasis adenocarcinoma & Temporal izquierdo \\
\hline 26 & M & 66 & Glioma alto grado & Parietooccipital izquierdo \\
\hline 27 & $\mathrm{H}$ & 28 & Glioma alto grado & Frontotemporal izquierdo \\
\hline 28 & $\mathrm{H}$ & 52 & Glioma alto grado & Temporal derecho \\
\hline 29 & $\mathrm{H}$ & 41 & Glioma alto grado & Temporal derecho \\
\hline 30 & M & 61 & Glioma alto grado & Temporal derecho \\
\hline
\end{tabular}




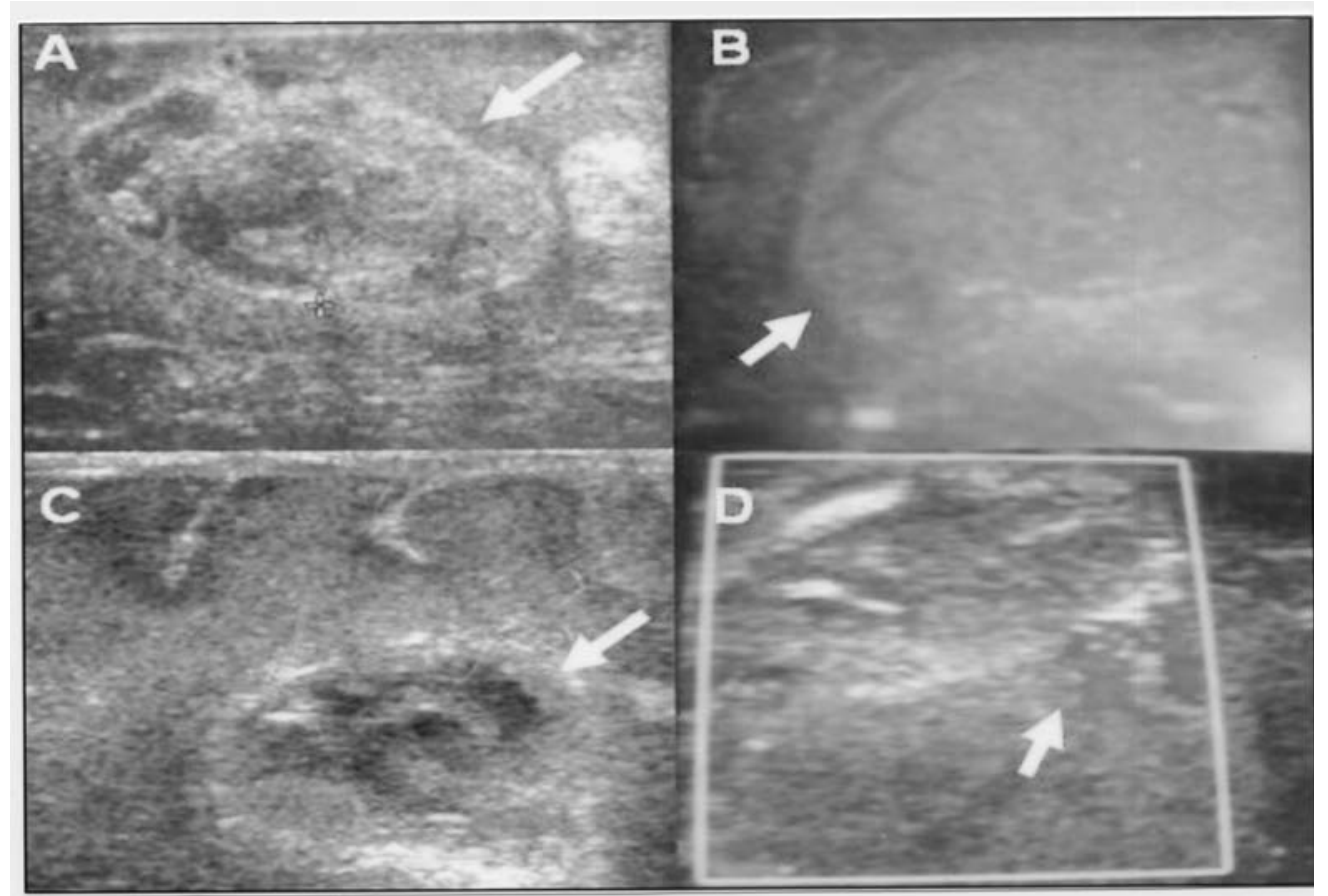

Figura 1. Imágenes por ultrasonografia intraoperatorias de lesiones tumorales pertenecientes a pacientes incluidos en nuestro estudio. $A$, metástasis cerebral de un adenocarcinoma pulmonar. B y C, gliomas de alto grado. $D$, vascularización en un tumor detectado con el uso de la ultrasonografía.

ción del tumor cerebral. Se consiguieron cortes en plano sagital y coronal durante la craneotomía parietal o temporal y en plano axial y sagital en la craneotomía frontal, para situar el área de interés en un campo cercano al transductor. Después de la exéresis del tumor, se realizó una nueva USIO tras irrigar la cavidad con suero salino para la visualización de remanente tumoral, los bordes de la cavidad y el tejido circundante. Se realizó una TC craneal en el periodo postoperatorio a todos los pacientes incluidos en el estudio para verificar la exéresis total del tumor.

\section{Resultados}

Se incluyeron en el estudio a 30 pacientes, 22 hombres y 8 mujeres, con edades comprendidas entre 28 y 82 años (media de $63 \pm 12$ ). El tiempo requerido para la localización del tumor, así como la profundidad de localización y el tamaño del tumor se muestran en la tabla 1 . Se realizaron 8 craneotomías temporales, 7 parietooccipitales, 8 parietales, 2 frontoparietales y 5 parietotemporales. En 13 pacientes el tumor se localizó en el hemisferio dominante y en 17 en el hemisferio no dominante. El estudio histológico reveló 17 gliomas de alto grado, 3 gliomas de bajo grado, 8 lesiones metastásicas y 2 oligoastrocitomas. Las características demográficas de los pacientes estudiados, así como la histología y localización del tumor se muestran en la tabla 2.

Los gliomas de bajo grado se mostraron como estructuras ecogénicas homogéneas fácilmente diferenciables del parénquima cerebral, así como los oligoastrocitomas. Sin embargo, las metástasis (figura 1-A) y los gliomas de alto grado (figuras 1-B y 1-C) mostraron mayor ecogeni- dad que los gliomas de bajo grado, siendo identificadas como lesiones malignas debido a las áreas de necrosis de baja ecogenidad y de edema peritumoral identificado como estructura hiperecogénica. En la figura 1-D podemos comprobar como la USIO nos permite identificar los vasos cerebrales relacionados con el tumor, que en muchos casos nos facilitaría la localización del mismo.

No se registraron complicaciones durante la exéresis del tumor en ninguno de los pacientes en relación con la técnica, así como no se detectaron complicaciones hemorrágicas ni déficit neurológico añadido. Asimismo, no se detectó restos de tumor al final de la cirugía, excepto en 2 pacientes debido a la proximidad a áreas elocuentes del cerebro. La realización de un TC craneal postoperatorio confirmó la exéresis total del tumor en el resto de los pacientes estudiados. En la figura $2 \mathrm{se}$ muestran las imágenes de ultrasonografía 2-D intraoperatoria y la TC craneal postoperatoria en un paciente intervenido para resección de un glioma de alto grado. La RM de cráneo preoperatoria muestra un proceso expansivo parietal derecho (figura 2-A). En la figura 2-B se puede apreciar la imagen por ultrasonidos de la lesión tumoral durante la craneotomía. La realización de una TC craneal postoperatoria reveló la exéresis total del tumor (figura 2-C). En dos pacientes fue imposible la exéresis total del tumor a pesar de la utilización de la USIO debido a la proximidad de la lesión a áreas elocuentes del cerebro, como es el caso que se muestra en la figura 3.

\section{Discusión}

Este estudio demuestra que la utilización de la USIO es eficaz para la localización de tumores cerebrales supra- 


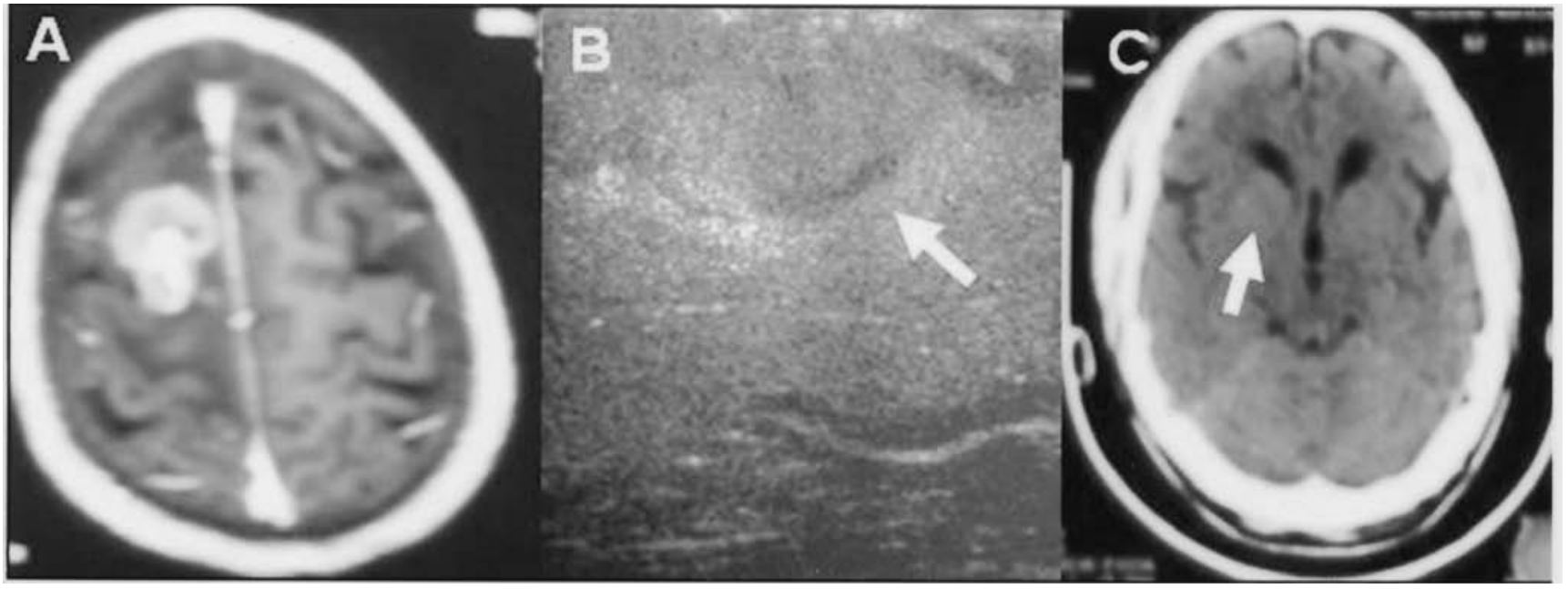

Figura 2. A, resonancia magnética preoperatoria que muestra glioma de alto grado parietal derecho. B, imagen por ultrasonografía intraoperatoria tras la apertura de la duramadre. C, tomografía computarizada craneal realizada en el postoperatorio donde no se aprecian restos tumorales.

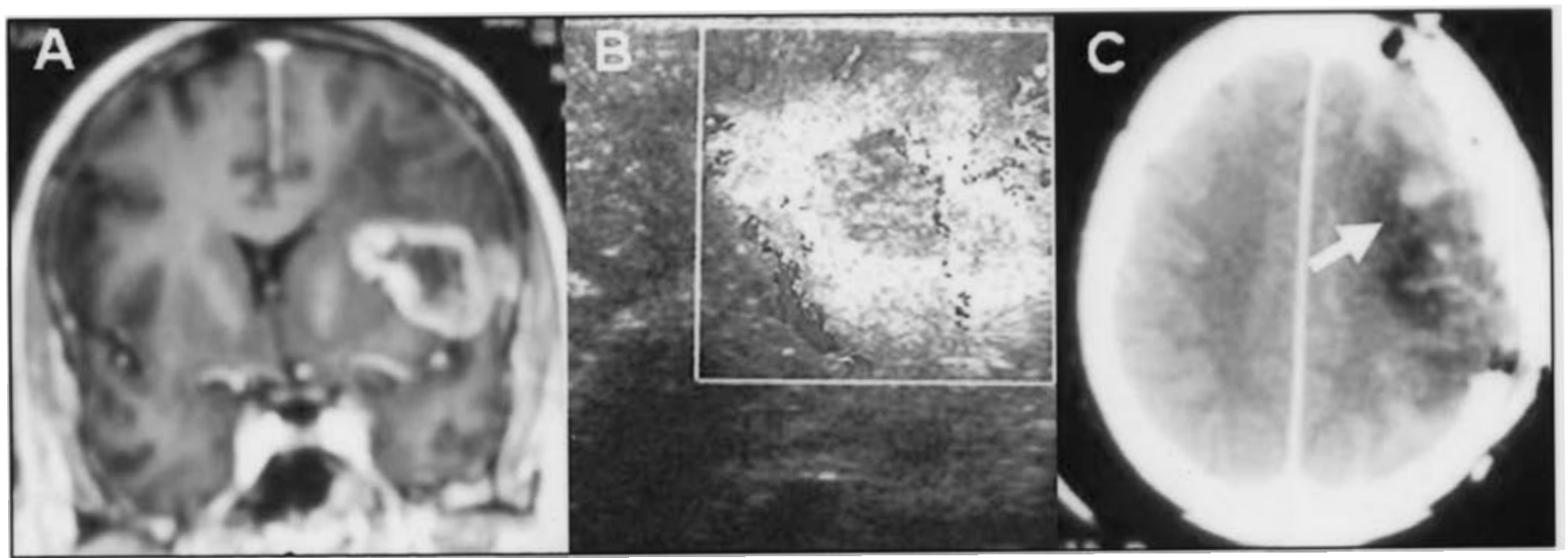

Figura 3. A, resonancia magnética preoperatoria que muestra glioma de alto grado frontal posterior izquierdo. B, imagen por ultrasonografía intraoperatoria tras la apertura de la duramadre. C, tomografía computarizada craneal realizada en el postoperatorio donde se aprecian restos en la parte medial de la lesión.

tentoriales, así como para detectar remanente de tumor tras la exéresis tumoral. En consecuencia con lo dicho y coincidiendo con otros autores, en nuestra serie de 30 pacientes localizamos el tumor durante la craneotomía con elevada precisión independiente del diagnóstico histopatológico ${ }^{7,12}$. De igual modo, fue posible la identificación de los borde del tumor antes de la resección en todos los pacientes incluidos en este estudio. En nuestra serie incluimos principalmente gliomas de alto grado y metástasis cerebrales que mostraron una imagen ultrasonográfica de alta ecogenidad en comparación con el cerebro sano. En cambio, los gliomas de bajo grado se mostraron como estructuras más homogéneas y de menor ecogenidad, con una pobre demarcación entre el tumor y el cerebro, que en ocasiones podría dificultar la exéresis tumoral ${ }^{8}$. No obstante, la incorporación de nuevos equipos de ultrasonografía con mayor poder de resolución asociado a la elevada experiencia y habilidad del neurorradiólogo, ha facilitado la exéresis en este tipo de tumor.

Asimismo, hemos podido comprobar que la USIO permite detectar la línea de demarcación entre el tumor y el cerebro, comprobando que los bordes de los gliomas de alto grado y de las metástasis son más densos e irregulares que en los gliomas de bajo grado, debido al carácter invasivo de los tumores malignos ${ }^{6-8}$. Asimismo, el componente necrótico se observa principalmente en los gliomas de alto grado y en las metástasis, con apariencia sonográfica de baja ecogenidad, localizado en el área central y paracentral 
del parénquima tumoral. Por otro lado, se ha constatado que las lesiones quísticas se muestran como áreas hipoecogénicas rodeadas de parte sólida tumoral o de estructuras membranosas. Las lesiones quísticas se asocian a cambios perilesionales cuando aparecen en tumores malignos, que incluye una banda hipoecogénica semejante al tejido cerebral, íntimamente unida al tumor, en la que están presentes lesiones isquémicas y cambios glióticos, que se continúa con una zona de edema vasogénico de ecogenidad similar a la parte sólida del tumor.

Diversos autores han demostrado en pacientes con tumor cerebral un aumento del tiempo de supervivencia relacionada con el grado de resección tumoral ${ }^{1,14}$. Wirtz et al. ${ }^{15}$ demostraron una prolongación significativa del tiempo de supervivencia tras la resección radical de gliomas con la utilización de la RM intraoperatoria. Por otra parte, Hammonu et al. ${ }^{7}$ y Woydt et al. ${ }^{16}$, indicaron que la USIO podría ser una modalidad de imagen alternativa a la RM intraoperatoria para la craneotomía guiada en diferentes procedimientos neuroquirúrgicos, ya que los ultrasonidos proporcionan mejor información sobre la extensión y localización del tumor, facilitando la orientación del neurocirujano durante la exéresis del mismo. Coincidiendo con estos autores, Unsgaard et al. ${ }^{12}$, mostraron en su estudio resultados óptimos de la utilización de la USIO similares a los publicados por otros autores en relación a la RM intraoperatoria para el control de la resección tumoral en neurocirugía $a^{71}$. Sin embargo, las técnicas de imagen por medio de RM intraoperatoria suponen un coste elevado, por lo que no son asequibles para muchos hospitales, constituyendo la ultrasonografía una alternativa eficaz a un coste aceptable como técnica de imagen intraoperatoria.

Recientemente, ha sido introducido en neurocirugía los sistemas de navegación guiada por computador para facilitar el abordaje neuroquirúrgico y reducir la manipulación de áreas elocuentes del cerebro. Estos sistemas de neuronavegación ofrecen la ventaja de la localización precisa de la lesión permitiendo realizar una mínima craneotomía; sin embargo, puede orientar incorrectamente al neurocirujano cuando la resección de la lesión ha sido iniciada, ya que existen errores significativos debidos a desplazamientos del cerebro por pérdidas de líquido cefalorraquídeo tras la apertura de la duramadre ${ }^{4}$.

La principal limitación de la utilización de la USIO para la exéresis de tumor cerebral es la excesiva reverberación presente en las imágenes obtenidas que pueden dificultar la información acerca de estructuras anatómicas y lesiones cerebrales profundas, y complicar la orientación del neurocirujano durante la resección tumoral. Por lo tanto el beneficio de los USIO como guía de procedimientos neuroquirúrgicos es dependiente de la experiencia del neurorradiólogo y de la posición y tamaño de la craneotomía. En la actualidad se está utilizando en neurocirugía la ultrasonografía tridimensional integrada con la tecnología de la navegación para el control de la resección de tumores cerebral, ya que se ha demostrado soluciona el problema de la orientación ${ }^{13}$. Sin embargo, este sistema no dispone de la capacidad de imagen en tiempo-real de la ultrasonografía que proporcione un grado de seguridad satisfactorio durante la craneotomía guiada durante la cirugía tumoral.

Según nuestra experiencia clínica, la ultrasonografía 2$D$ en tiempo-real proporciona una imagen de calidad aceptable para la realización de craneotomía guiada para exéresis de tumores supratentoriales, ya que permite su localización de manera fiable y segura, sobre todo en tumores localizados en zonas del cerebro con gran importancia anatómica y funcional, así como identificar restos de tumor una vez finalizada la cirugía. Asimismo, la USIO es un método coste/efectivo, rápido y de fácil manejo para neurocirugía. Por todo ello, recomendamos la ultrasonografía como procedimiento de rutina durante la craneotomía para exéresis de tumor supratentorial.

\section{Bibliografía}

1. Campbell, J.W., Pollack, I.F., Martinez, A.J., Shultz, B.: High-grade astrocitomas in children: Radiologically complete resection is associated with an excellent long-term prognosis. Neurosurgery 1996; 38: 258-264.

2. Chandler, W.F., Knake, J.E., McGillicuddy, J.E., Lillehei, K.O., Silver, T.M.: Intraoperative use of real-time ultrasonography in neurosurgery. J Neurosurg 1982; 57: 157-163.

3. Dormán, G.J., Rubin, J.M.: History of intraoperative ultrasound in Neurosurgery. Neurosurg Clin N Am 2001; 12: 155-166.

4. Dorward, N.L., Alberti, O., Velani, B., et al.: Postimaging brain distortion: magnitudes, correlates and impact on neuronavegation. J Neurosurg 1998; 88: 656-662.

5. Grode, M.L., Komaiko, M.S.: The role of intraoperative ultrasound in neurosurgery. Neurosurgery 1983; 12: 624-628.

6. Gronningsaeter, A., Unsgaard, G., Ommedal, S, Angelsen, B.A.: Ultrasound-guide neurosurgery. A feasibility study in the $3-30 \mathrm{MHz}$ frequency range. $\mathrm{Br} \mathrm{J}$ Neurosurg 1996; 10: 161-168.

7. Hammoud, M.A., Ligon, B.L., elSouki, R., Shi, W.M., Schomer, D.F., Sawaya, R.: Use of intraoperative ultrasounds for localizing tumors and determining the extent of resection: a comparative study with magnetic resonance imaging. J Neurosurg 1996; 84: 737-741.

8. Knake, J.E., Chandler, W.F., Gabrielzen, T.O., Latack, J.T., Gabarski, S.S.: Intraoperative sonographie delineation of low grade brain neoplasm defined poorly by computed tomography. Radiology 1984; 151; 735-739.

9. Regelsberger, J., Lohmann, F., Helmke, K., Westphal, M.: Ultrasound-guided surgery of deep seated brain lesions. 
Eur J Ultrasound 2000; 12: 115-121.

10. Rubin, J.M., Mirfakhraee, M., Duda, E.E., Dohrmann, G.J., Brown, F.: Intraoperative ultrasound examination of the brain. Radiology 1980; 137: 831-832.

11. Sutcliffe, J.C.: The value of intraoperative ultrasound in neurosurgery. Br J Neurosurg. 1991; 5: 169-178.

12. Unsgaard, G., Gronningsaeter, A., Ommedal, S., Nagelhus Hernes, T.A.: Brain operations guided by real-time two-dimensional ultrasound: New possibilities as a result of improved image quality. Neurosurgery 2002; 51: 402-412.

13. Unsgaard, G., Ommedal, S., Muller, T., Gronningsaeter, A., Nagelhus Hernes, T.A.: Neuronavegation by intraoperative three-dimensional ultrasound: Initial experience during brain tumor resection. Neurosurgery 2002; 50: 804-812.

14. Wirtz, C.R., Albert, F.K., Schwaderer, M., et al.: The benefit of neuronavegation for neurosurgery analyzed by its impact on Glioblastoma surgery. Neurol Res 2000; 22: 354360.
15. Wirtz, C.R., Knauth, M., Staubert, A., Bonsanto, M.M., Sartor, K., Kunze, S., Tronnier, V.M.: Clinical evaluation and follow-up results for intraoperative magnetic resonance imaging in neurosurgery. Neurosurgery 2000; 46: 1112-1122.

16. Woydt, M., Krone, A., Becker, G., Schmidt, K., Roggendorf, W., Roosen, K.: Correlation of intra-operative ultrasound with histopathologic findings after tumor resection in supratentorial gliomas. A method to improve gross total tumor resection. Acta Neurochir (Wien) 1996; 138: 1391-1398.

López-Hernández, F.; Hernández-Palazón, J.; ReusPintado, M.; Garrido-Gómez, J.I.; Martínez-Lage, J.F.: Craneotomía guiada por ultrasonografía bidimensional para exéresis de tumor cerebral supratentorial. Neurocirugía 2008; 19: 530-536.

Correspondencia postal: Joaquín Hernández Palazón. C/ San Ignacio de Loyola, 4-2 ${ }^{\circ}$ B. 30001 Murcia. 